\title{
Deciphering Bacterial Community Structure, Functional Prediction and Food Safety Assessment in Fermented Fruits Using Next-Generation 16S rRNA Amplicon Sequencing
}

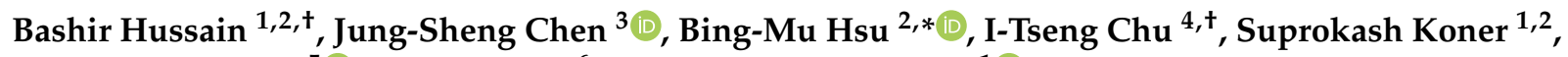 \\ Tsung-Hsien Chen ${ }^{5}{ }^{\mathbb{D}}$, Jagat Rathod ${ }^{6}$ and Michael W. Y. Chan ${ }^{1}{ }^{-1}$ \\ 1 Department of Biomedical Sciences, National Chung Cheng University, Chiayi 621, Taiwan; \\ bashir.aku@gmail.com (B.H.); suprokashkoner22@gmail.com (S.K.); biowyc@ccu.edu.tw (M.W.Y.C.) \\ 2 Department of Earth and Environmental Sciences, National Chung Cheng University, Chiayi 621, Taiwan \\ 3 Department of Medical Research, E-Da Hospital, Kaohsiung 824, Taiwan; nicky071214@gmail.com \\ 4 Department of Internal Medicine, Division of Cardiology, Ditmanson Medical Foundation Chia-Yi Christian \\ Hospital, Chiayi 621, Taiwan; 02500@cych.org.tw \\ 5 Department of Internal Medicine, Ditmanson Medical Foundation Chia-Yi Christian Hospital, \\ Chiayi 621, Taiwan; cych13794@gmail.com \\ 6 Department of Earth Sciences, National Cheng Kung University, 1st University Road, Tainan 701, Taiwan; \\ jagat2006@gmail.com \\ check for \\ updates \\ Citation: Hussain, B.; Chen, J.-S.; \\ * Correspondence: bmhsu@ccu.edu.tw; Tel.: +886-5272-0411 (ext. 66218) \\ + Equal contribution to this study.
} Hsu, B.-M.; Chu, I-T.; Koner, S.; Chen, T.-H.; Rathod, J.; Chan, M.W.Y. Deciphering Bacterial Community Structure, Functional Prediction and Food Safety Assessment in Fermented Fruits Using Next-Generation 16S rRNA Amplicon Sequencing. Microorganisms 2021, 9 , 1574. https://doi.org/10.3390/ microorganisms 9081574

Academic Editors: Vincenzina Fusco, Hikmate Abriouel and Evandro Leite de Souza

Received: 21 May 2021

Accepted: 22 July 2021

Published: 23 July 2021

Publisher's Note: MDPI stays neutral with regard to jurisdictional claims in published maps and institutional affiliations.

Copyright: (c) 2021 by the authors. Licensee MDPI, Basel, Switzerland. This article is an open access article distributed under the terms and conditions of the Creative Commons Attribution (CC BY) license (https:/ / creativecommons.org/licenses/by/ $4.0 /)$.

\begin{abstract}
Fermented fruits and vegetables play an important role in safeguarding food security world-wide. Recently, robust sequencing-based microbial community analysis platforms have improved microbial safety assessment. This study aimed to examine the composition of bacteria and evaluate the bacterial safety of fermented fruit products using high-throughput 16S-rRNA metagenomic analysis. The operational taxonomic unit-based taxonomic classification of DNA sequences revealed 53 bacterial genera. However, the amplicon sequencing variant (ASV)-based clustering revealed 43 classifiable bacterial genera. Taxonomic classifications revealed that the abundance of Sphingomonas, which was the predominant genus in the majority of tested samples, was more than $85-90 \%$ among the total identified bacterial community in most samples. Among these identified genera, 13 low abundance genera were potential opportunistic pathogens, including Acinetobacter, Bacillus, Staphylococcus, Clostridium, Klebsiella, Mycobacterium, Ochrobactrum, Chryseobacterium, Stenotrophomonas, and Streptococcus. Of these 13 genera, 13 major opportunistic pathogenic species were validated using polymerase chain reaction. The pathogens were not detected in the samples of different stages and the final products of fermentation, except in one sample from the first stage of fermentation in which S. aureus was detected. This finding was consistent with that of ASV-based taxonomic classification according to which $S$. aureus was detected only in the sample from the first stage of fermentation. However, S. aureus was not significantly correlated with the human disease pathways. These results indicated that fermentation is a reliable and safe process as pathogenic bacteria were not detected in the fermentation products. The hybrid method reported in this study can be used simultaneously to evaluate the bacterial diversity, their functional predictions and safety assessment of novel fermentation products. Additionally, this hybrid method does not involve the random detection of pathogens, which can markedly decrease the time of detection and food safety verification. Furthermore, this hybrid method can be used for the quality control of products and the identification of external contamination.
\end{abstract}

Keywords: fermented fruits; bacterial diversity; food safety; functional prediction; next-generation sequencing; opportunistic pathogens; $16 \mathrm{~S}$ rRNA metagenomics 


\section{Introduction}

Fermentation is a cost-effective method to preserve perishable and seasonal fruits, which play a vital role in safeguarding food security world-wide [1,2]. Fermented products, which have several advantages, including increased nutritional value, are a major source of minerals, vitamins, and carbohydrates to the vulnerable population [3,4]. Moreover, fermented products of fruits and vegetables are an integral part of most cuisines worldwide [5]. Various types of fermented foods are estimated to comprise up to $30 \%$ of the food supply [6]. However, the microbiological safety of these products must be ensured before public consumption [7]. Generally, fermented products are considered microbiologically safe as the harsh fermentation conditions, such as low $\mathrm{pH}$, high temperature, and increased sugar and salt contents prevent the growth of pathogenic microbes [6,8]. However, several studies have demonstrated that some pathogens, such as Listeria monocytogenes, Clostridium spp., Salmonella spp., Staphylococcus spp., Bacillus spp., and Escherichia coli survive and escape during fermentation and remain infectious [9-12]. The fermentation conditions, such as the time-temperature profile, water activity, and $\mathrm{pH}$ determine the microbial profile of the fermented products $[13,14]$. Additionally, other factors, including poor sanitation, starter culture quality, and raw material quality, contribute to the microbial profiles of fermented food [14,15].

Recently, there is increased consumer awareness on food safety and health benefits, including food quality and microbial traceability and safety [16,17]. The recent advances in next-generation sequencing (NGS) have revolutionized the traceability and characterization of a broad range of pathogens in various fields, including epidemiology, diagnostics, food quality, and forensics $[18,19]$. The fermentation process involves nonfunctional microbial communities, active microbial communities, and abiotic factors, which decrease the emergence of pathogenic microbes during the fermentation processes and the final products $[14,19]$. Some bacteria, especially the members of Enterococcus and Enterobacteriaceae, produce toxic metabolites, such as biogenic amines during the fermentation process [19]. Thus, the consumption of food products containing toxic metabolites and viable pathogens may lead to various foodborne illnesses [19]. Some fermented food-associated microorganisms, such as Enterococcus, Staphylococcus, and Lactobacillaceae can harbor transmissible antibiotic resistance genes [20] Hence, there is a need to characterize the function and diversity of microbes in fermented food products to improve the quality and safety measures of the final product [21]. Traditional approaches, such as biochemical and physiological methods used for routine enumeration and characterization of pathogens are expensive, laborious, and time-consuming [22,23]. Additionally, traditional methods cannot provide an accurate pathogen risk assessment as they cannot capture the complete microbial diversity [24]. Molecular approaches based on the DNA/RNA signatures of food matrices enable the understanding of microbial complexity and the ecology of the fermentation process at the molecular level. Additionally, molecular approaches can capture the complete microbial population, including the viable but non-culturable microbes $[25,26]$. Furthermore, molecular approaches can detect injured or stressed microbial populations [27]. The advances in NGS-based technologies have decreased the cost of microbial analysis and increased the resolution of microbial community analysis [28,29]. Recent studies have utilized NGS to successfully examine the microbial ecology of various fermentation processes of food items, such as tea, milk, wine, fruits, and vegetables [21,24,30,31]. However, most studies have focused on the characterization of functional and beneficial microbial diversity. There are limited studies examining the microbiological safety of fermentation products using NGS techniques [32].

This study aimed to investigate the total bacterial community and their functional prediction along with the identification of potential pathogens in fermented fruits at different stages of fermentation. In this study, a metagenomics approach was used to target the V3 and V4 regions of the $16 \mathrm{~S}$ rRNA gene to determine the change in bacterial diversity in the different stages of fermentation and the final fermentation products. The identification of unique bacterial communities, including pathogens, and their predicted functions can 
aid in improving food safety. This study characterized the bacterial community structures and their safety parameters, which can increase the consumer acceptance of fermented fruit-based products.

\section{Materials and Methods}

\subsection{Samples and Chemical Analysis}

In this study, sampling was performed during various stages of the fruit fermentation process using the intermediate and finished products from a commercial fermented beverage production company (Taiwan Enzyme Village Co., Ltd. Chiayi, Taiwan) located in Chiayi County, Taiwan. The fruit fermentation of pineapple, orange, and banana is carried out into two stages, which ultimately lead to the final product. In the 1st stage of fermentation, these fruits are washed and cut in fermentation tanks followed by the addition of sugar, yeast, lactic acid bacteria (LAB), and acetic acid bacteria (AAB), then followed by mixing vigorously for $24 \mathrm{~h}$. The concentration of yeast, LAB, and AAB additives was $0.0025 \%$, whereas, the concentration of sugar was $23.2 \%$ in a total of 1 metric ton of fermented fruit. However, pineapple samples without starter culture did not contain yeast, $\mathrm{LAB}$, and $\mathrm{AAB}$ in the fermentation tanks. Finally, these fermentation tanks were kept at room temperature under anaerobic conditions to accomplish the 1st stage of the fermentation process. The total fermentation duration of pineapple, banana and orange in the 1st stage was approximately 180, 150, and 365 days, respectively. The sampling time was based on $1 / 3$ and $2 / 3$ of the fermentation duration for pineapple and banana, whereas orange was collected at $1 / 2$ and $3 / 4$ of the fermentation duration. The 2 nd stage of fermentation was performed by mixing the above fermented products from the 1st stage in a fermentation tank and kept for approximately one year at room temperature under anaerobic conditions. Real-time temperature detection was used to ensure the range of room temperature (in between $24-28{ }^{\circ} \mathrm{C}$ ) during fermentation. The final product of fermentation was a mixture of liquid products from the 2nd stage of fermentation. In total, 28 samples ( $40 \mathrm{~mL}$ of fermented broth for each sample) were aseptically collected from their respective fermentation tanks and final product into sterilized containers and transported to the laboratory under controlled temperature. Description of the samples, fermentation processes and sampling time are provided in Table 1. Additionally, a brief description of chemical analysis from the 2nd stage of fermentation and the final product are shown in Supplementary Table S2.

Table 1. Description of samples and fermentation processes based on raw materials and fermentation duration.

\begin{tabular}{|c|c|c|c|}
\hline Sample ID & Full Name & Process & Fermentation Sampling Time \\
\hline Or1_1st & Orange-0120 & \multirow{14}{*}{$\begin{array}{l}\text { 1st stage of } \\
\text { fermentation }\end{array}$} & \multirow{3}{*}{208 day } \\
\hline Or2_1st & Orange-1272 & & \\
\hline Or3_1st & Orange-1266 & & \\
\hline PiBac1_1st & Pineapple w/starter culture-0099 & & \multirow{3}{*}{67 day } \\
\hline PiBac2_1st & Pineapple w/starter culture-0183 & & \\
\hline PiBac3_1st & Pineapple w/starter culture-0151 & & \\
\hline Pi1_1st & Pineapple w/o starter culture-0912 & & \multirow{2}{*}{60 day } \\
\hline Pi2_1st & Pineapple w/o starter culture- 0080 & & \\
\hline Ba1_1st & Banana-0174 & & \multirow{3}{*}{45 day } \\
\hline Ba2_1st & Banana-0245 & & \\
\hline Ba3_1st & Banana-1267 & & \\
\hline Or4_1st & Orange-0120 & & \multirow{3}{*}{268 day } \\
\hline Or5_1st & Orange-1272 & & \\
\hline Or6_1st & Orange-1266 & & \\
\hline
\end{tabular}


Table 1. Cont.

\begin{tabular}{|c|c|c|c|}
\hline Sample ID & Full Name & Process & Fermentation Sampling Time \\
\hline PiBac4_1st & Pineapple w/starter culture-0099 & & \multirow{3}{*}{127 day } \\
\hline PiBac5_1st & Pineapple w/starter culture-0183 & & \\
\hline PiBac6_1st & Pineapple w/starter culture-0151 & & \\
\hline Pi3_1st & Pineapple w/o starter culture-0242 & & \multirow{3}{*}{127 day } \\
\hline Pi4_1st & Pineapple w/o starter culture-0912 & & \\
\hline Pi5_1st & Pineapple w/o starter culture-0080 & & \\
\hline Ba4_1st & Banana-0174 & & \multirow{2}{*}{105 day } \\
\hline Ba5_1st & Banana-1267 & & \\
\hline Ch1_2nd & 1st stage product No.24 & \multirow{3}{*}{$\begin{array}{l}\text { 2nd stage of } \\
\text { fermentation }\end{array}$} & \multirow{3}{*}{240 day } \\
\hline Ch2_2nd & 1st stage product No.28 & & \\
\hline Ch3_2nd & 1st stage product No.30 & & \\
\hline Pr1_F & Product No.1 & \multirow{3}{*}{ Product } & \multirow{3}{*}{0 day * } \\
\hline Pr2_F & Product No.2 & & \\
\hline Pr3_F & Product No.3 & & \\
\hline
\end{tabular}

\subsection{Genomic DNA (gDNA) Extraction and Bacterial $16 S$ rRNA Amplicon Analysis}

The fermented broth $(2 \mathrm{~mL})$ was thoroughly mixed, aseptically transferred to a sterilized tube, and centrifuged at $12,000 \times g$ for $3 \mathrm{~min}$. The supernatant was discarded and the bacterial cells were lysed to extract gDNA using a commercial auto DNA extractor kit (MagPurix Bacterial DNA Extraction Kit, ZP02006, Taipei, Taiwan), following the manufacturer's instructions. The purity and concentration of the extracted gDNA were determined using a Nanodrop 2000 spectrophotometer (Thermo Fisher Scientific Inc., Wilmington, DE, USA) at $230-280 \mathrm{~nm}$. The quality of gDNA was examined using gel electrophoresis (1.5\% gel in Tris-acetate ethylenediaminetetraacetic acid buffer) at $110 \mathrm{~V}$ for $30 \mathrm{~min}$. DNA bands were visualized under ultraviolet light. The purified gDNA was stored at $-20{ }^{\circ} \mathrm{C}$ for further analysis.

\subsection{Sequencing, Library Construction, and $16 S$ rRNA Amplicon Data Analysis}

The libraries for the amplified V3-V4 regions of 16S rRNA hypervariable regions were constructed using the $341 \mathrm{~F}$ and $805 \mathrm{R}$ primer sets (with some modifications) with the Illumina adapter overhang sequence attached at the $5^{\prime}$ end of the primers. The sequences of forward primers used in this experiment were as follows: (16S_341F) $5^{\prime}$ TCGTCGGCAGCGTCAGATGTGTATAAGAGACAGCCTACGGGNGGCWG CAG-3', (16S_341F_N) 5'-TCGTCGGCAGCGTCAGATGTGTATAAGAGAC AGnCCTACGGGNGG CWGCAG-3', (16S_341F_N N) 5'-TCGTCGGCAGCGTCAG ATGTGTATAAGAGACAGnnCCTACGGGNGGC WGCAG-3' ${ }^{\prime}$, and (16S_341F_NNN) $5^{\prime}$-TCGTCGGCAGCGTCAGATG TGTATAAGAGACAGnnnCCTACGGGNGG CWGCAG-3'. The reverse primer sequences were as follows: (16S_805R) 5'-GTCTCGTGGGCTCGGAGATGTGTATAAGAGACAGGAC TACHVGGGTA TCT AATCC-3', (16S_805R_N_) 5'-GTCTCGTGGGCTCGGAGATGTGTA TAAGAGA CAGnGACTACHVGGGTATCTAATCC-3', (16S_805R_NN) 5'-GTCTCGTG GGC TCGGAGATGTGTATAAGAGACAGnnGACTACHVGGGTATCTAATCC $-3^{\prime}$, and (16S_805R_NNN) 5'-GTCTCGTGGGCTCGGAGATGTGTATAAGAGACAG nnnGACTAC HVGGGTATCTAATCC $-3^{\prime}$. The amplification was performed in triplicates, following the previously reported method [33]. The optimal PCR conditions were as follows: $95^{\circ} \mathrm{C}$ for $3 \mathrm{~min}$, followed by 30 cycles of $95^{\circ} \mathrm{C}$ for $30 \mathrm{~s}$ (denaturation), $55^{\circ} \mathrm{C}$ for $30 \mathrm{~s}$ (annealing), $72{ }^{\circ} \mathrm{C}$ for $30 \mathrm{~s}$ (primer extension), and $72{ }^{\circ} \mathrm{C}$ for $5 \mathrm{~min}$ (elongation). The quantity and quality of amplified DNA were assessed using the standard quality checks mentioned above. Next, 
the amplicons $(20 \mu \mathrm{L})$ from each sample were subjected to sequencing using the pair-end method with the MiSeq Illumina platform (Illumina Inc., San Diego, CA, USA), following standard protocol at the National Yang-Ming University Genome Research Center. The DNA libraries were ligated with the sequencing adapters and index using the Nextera XT sample preparation kit (Illumina), following the manufacturer's instructions. The sequence data containing reverse and forward reads were aligned using CLC bio plate form (Genomic Workbench v.8.5) and the FASTA files were generated as described in our previous study [34]. The FASTA files were further processed using two standard 16S-metagenomics pipelines. The USEARCH system was used to remove chimeric sequences. The sequences were clustered into operational taxonomic units (OTUs) with a 97\% similarity threshold against the Green Genes database. The QIIME2 system was used for the sequence quality control and amplicon sequence variant (ASV)-based classification [35]. Finally, the relative abundance of microbes at the genus level in each sample was obtained using the QIIME2 view. Furthermore, the significant difference in the relative abundance at the genus level at each fermentation stage and in the final fermentation product was analyzed using the statistical analysis of taxonomic and functional profiles (STAMP) software [36]. The significant relative abundance at the genus levels was examined using the two-tailed Welch's t-test $(p<0.05)$.

\subsection{Metagenomic Functional Prediction Based on $16 S$ rRNA Gene Data}

To examine the potential metabolic function of microorganisms at different stages of fermentation, the representative sequence and denoised ASV abundance table were used. These tables were input into the phylogenetic investigation of communities' reconstruction of Unobserved States (PICRUSt2) pipeline (https:/ / github.com/picrust/picrust2/, accessed on 15 November 2020) using KEGG (Kyoto Encyclopedia of Genes and Genomes). All ASVs with nearest-sequenced taxon index (cut-off value $>2$ ) were removed by default for reliable annotation of metabolic functions using the KEGG reference database as previously described [37]. To improve the accuracy and reliability of the KEGG pathways, a previously described web-based tool (MicrobiomAnalyst) was used to remove extremely low abundant and variant KEGG gene orthologs (KOs) (based on $<4 \mathrm{KO}$ filtration) from each sample [38]. The results of levels 1 and 2 KEGG pathways were plotted in the R environment using the ggplot2 package. The significant shift in the bacterial community at each fermentation stage and fermentation product was analyzed using two-tail Welch's t-test with STAMP software $(p<0.05)$ with 95\% confidence intervals. Pearson correlation analysis was performed using IBM SPSS Statistics 24 (IBM, Armonk and North Castle, NY, USA) to evaluate the significant correlations between bacterial diversity and potential functional prediction considering $p$ ranging $0.01-0.05$.

\subsection{Detection Methods for Suspected Pathogens after 16S-rRNA Gene Surveys}

In this study, species identification of suspected pathogens in fermented samples were validated by various specific genes by targeting PCRs. Acinetobacter baturnannii was validated using intergenic spacer (ITS) PCR analysis as described previously [39]. Pseudomonas aeruginosa, Stenotrophomonas maltophilia, Gardmerella vaginalis, and Chryseobacterium indologenes were validated using 6S-23S ribosomal DNA region PCR analysis as described previously [40-43]. The specific targeting nuc, bal, recA, tyrB and pneumolysin genes PCRs were used to validate Staphylococcus aureus, Bacillus cereus sensu lato s.l, Ochrobactrum anthropic, Klebsiella pneumoniae, and Streptococcus pneumoniae, respectively [44-48]. Clostridium difficile was validated by two genes $(t c d A, t c d B)$ PCRs [49]. The conditions for the PCR amplification protocols are described in Supplementary Table S1. 


\section{Results}

3.1. Sequence Depth and Bacterial Biodiversity in the Fermented Fruit Samples with Respect to Fermentation Processes Based on 16S rRNA Gene Amplicons

The 16S rRNA amplicon sequencing targeting the V3 and V4 regions was performed to examine the bacterial community diversity and composition in each fermentation stage sample. After quality filtering and the removal of chimeric sequences, a total of 785,339 sequence reads (average 28,047 per sample) ranging from 4595-75,976 were obtained from 28 samples (Supplementary Figure S1A) and the rarefaction of these sequences was performed at the lowest sequence depth (4595) to compare bacterial diversity among the fermentation stages and final products (Supplementary Figure S1B). The average good's coverage was more than $99 \%$ at this sequencing depth, indicating enough resolution to analyze the downstream analysis such as alpha and beta diversity estimations (Supplementary Table S3).

The bacterial diversity and richness among the fermentation stages and final product was elevated using various alpha diversity indices as shown in Figure 1. Among the alpha diversity indices, the Observed (Figure 1A), Chao1 (Figure 1B), and ACE (Figure 1C) revealed higher diversity richness associated with the 1st stage of fermentation and a decrease diversity was observed with increasing the fermentation duration. However, none of these indices showed significance diversity richness among the fermentation stages and final products. Additionally, Simpson (Figure 1D) and Shannon (Figure 1E) alpha diversity indices revealed higher diversity abundance associated with the 2nd stage followed by 1st stage compared to final product of fermentation. However, only Simpson alpha diversity index showed significance among the fermentation stages and final products.
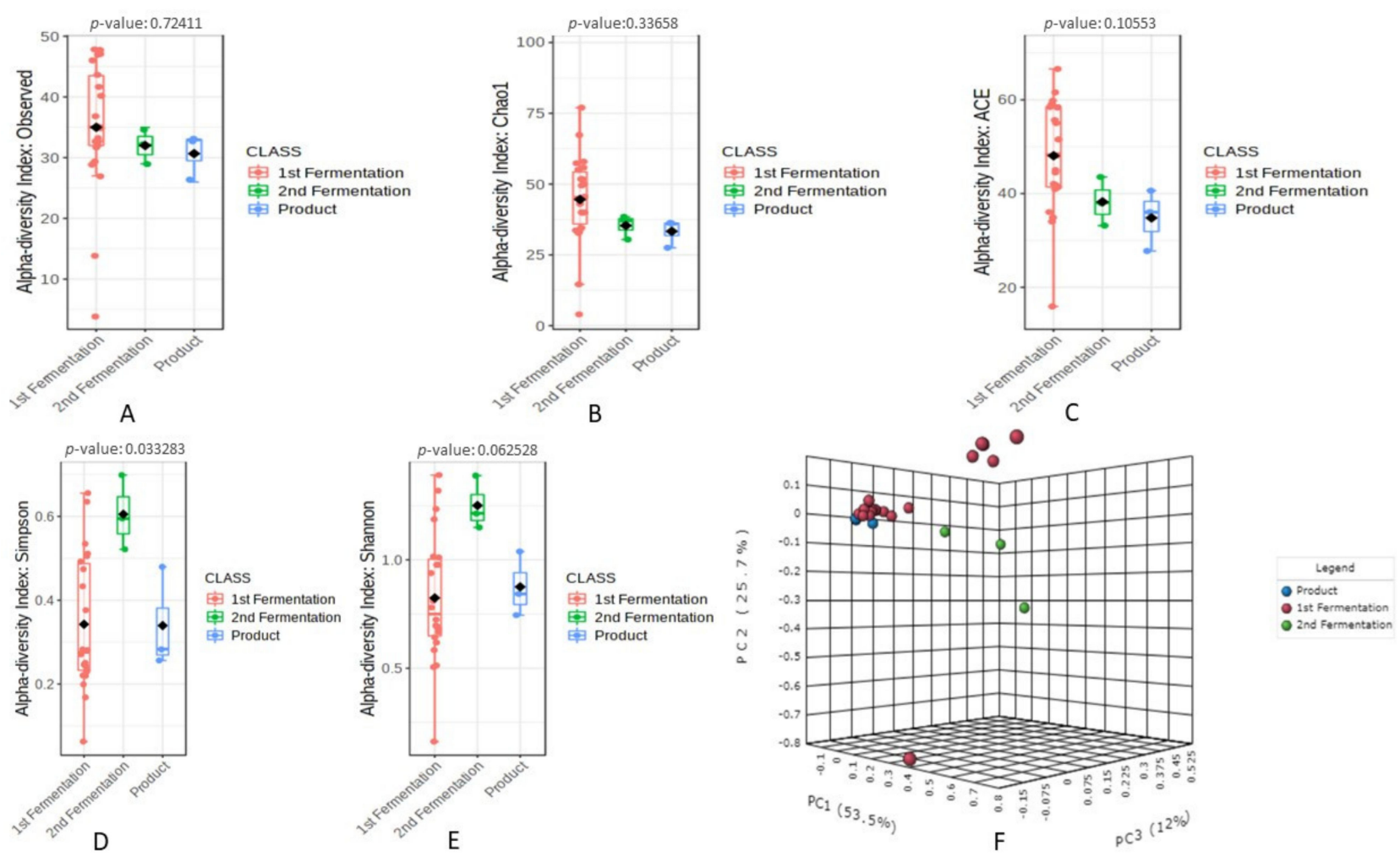

Figure 1. Comparison of bacterial community diversity among the fermentation stages and final product. Alpha diversity was measured by Observed (A), Chao1 (B), ACE (C), Simpson (D), and Shannon (E) diversity indices whereas, beta diversity was evaluated using PCoA (3D) ordination (F).

The beta diversity analysis based on Bray-Curtis distances revealed high variation among the bacterial diversity associated with the fermentation stages and product as the result showed a distinct clustering pattern (Figure 1F) indicating different bacterial 
communities among the experimental groups. The PC1 of ordination representing the 1st stage of fermentation showed the highest variation followed by the second stage of fermentation (PC2: $25.7 \%$ ) as compared to the final product (PC3: $12 \%$ ), which is inconsistent with the result of alpha diversity. Additionally, PARMANOVA was applied to know the significant analysis among the fermentation stages and final product, which showed significant variation $(p<0.05)$ in the bacterial beta diversity among the fermentation stages and final product.

\subsection{Bacterial Taxonomic Abundance in the Fermented Fruit Samples Evaluated Based on $16 S$ rRNA Gene Amplicons}

Genus level OTU based classification revealed a total of 52 classifiable genera as shown in Figure 2. Among them Sphingomonas was the most predominant bacteria, which accounted for more than $80 \%$ of the total bacteria, in most fermentation samples. The second most abundant bacterial genus was Oribacterium (18\%), which was the predominant genus in Ch3_2nd and Ba5_1st samples with maximum relative abundances of $>42 \%$ and $>98 \%$, respectively. The resolution of low abundance genera, which accounted for less than 10\% of the total genera, was limited due to the increased relative abundance of Sphingomonas. Therefore, this genus was excluded to visualize and interpret the relative abundance of the remaining 51 genera (Figure 2B). Of the 51 genera, 11 were potential opportunistic pathogens (Acinetobacter, Bacillus, Staphylococcus, Dietzia, Clostridium, Klebsiella, Mycobacterium, Ochrobactrum, Chryseobacterium, Stenotrophomonas, and Streptococcus). Among these pathogenic bacteria, Acinetobacter exhibited a high abundance and was detected in all samples. Bacillus, which was detected in $99 \%$ of samples, was the second most predominant genus. Stenotrophomonas, Streptococcus, and Staphylococcus were detected in more than 95\% of samples irrespective of their relative abundance. In contrast, Clostridium was detected in almost $67 \%$ of samples. Klebsiella, Chryseobacterium, Ochrobactrum, Mycobacterium, and Deutzia were detected in more than $10 \%$ of samples. Among the 28 samples, Ba1_1st and Ba3_1st exhibited a high distribution of bacterial genera with 15 genera detected in each sample.

To compare the bacterial diversity in each fermentation sample, the Qiime2 pipeline was used. After quality filtering, 193,323 sequence reads were obtained, which were classified into 43 identifiable genera (Figure 2C). Sphingomonas spp., which accounted for $85 \%$ of all bacteria was the most predominant bacterial genus in $98 \%$ of samples. The second most predominant bacteria were Nesterenkonia and Lactobacillus with maximum relative abundances of more than 3\%. Acetobacter (2.9\%) was the third most abundant genus, which was predominant in the Ba_1st sample and accounted for more than $85 \%$ of bacteria in the sample. Similarly, Lactobacillus (more than 65\%) was the predominant genus in the Pi1_1st sample. Among the potential opportunistic pathogens, the relative abundance of Bacillus, Clostridium, Dietzia, Gardnerella, and Pseudomonas was less than 1\% (Figure 2D). Dietzia and Bacillus were detected in $25 \%$ and $17 \%$ of samples, respectively. Clostridium, Gardnerella, and Pseudomonas were detected in less than 1\% of samples. Additionally, Actinomyces, Agrobacterium, Chryseobacterium, Clostridium, Dietzia, Methylobacterium, and Natromonsas were only present in pineapple samples containing starter culture (LAB and AAB) whereas, these bacteria were absent in non-starter culture containing pineapple samples. Contrarily, Achromobacter, Anaerobacillus, Delftia, Kocuria, Ochrobactrum, and Staphlococcus were only present in non-starter culture containing pineapple samples and these bacteria were absent in starter culture containing pineapple samples. Two genera including Lactobacillus and Nesterenkonia showed higher abundance in starter culture containing pineapple samples whereas, Sphingomonas abundance was higher in non-starter containing pineapple samples.

Both OTU and ASV taxonomic classifications generated a total of 13 opportunistic pathogenic genera associated with the fermentation processes and products, as shown in Table 2. Among these pathogenic genera, the ASV-based clustering approach identified Gardnerella and Pseudomonas, whereas the OTU-based clustering approach identified Acinetobacter, Klebsiella, Mycobacterium, and Streptococcus. All these bacteria were detected only in the samples from the first stage of fermentation, except Acinetobacter, which was detected 
in the samples from the first stage of fermentation and the final fermentation product. Other pathogenic genera commonly identified in both clustering approaches included Bacillus, Staphylococcus, Clostridium, Ochrobactrum, Cryseobacterium, Dietzia, and Stenophomonas, which were detected only in the samples from the first and second fermentation stages, whereas, Bacillus and Clostridium were also detected from the final fermentation products.
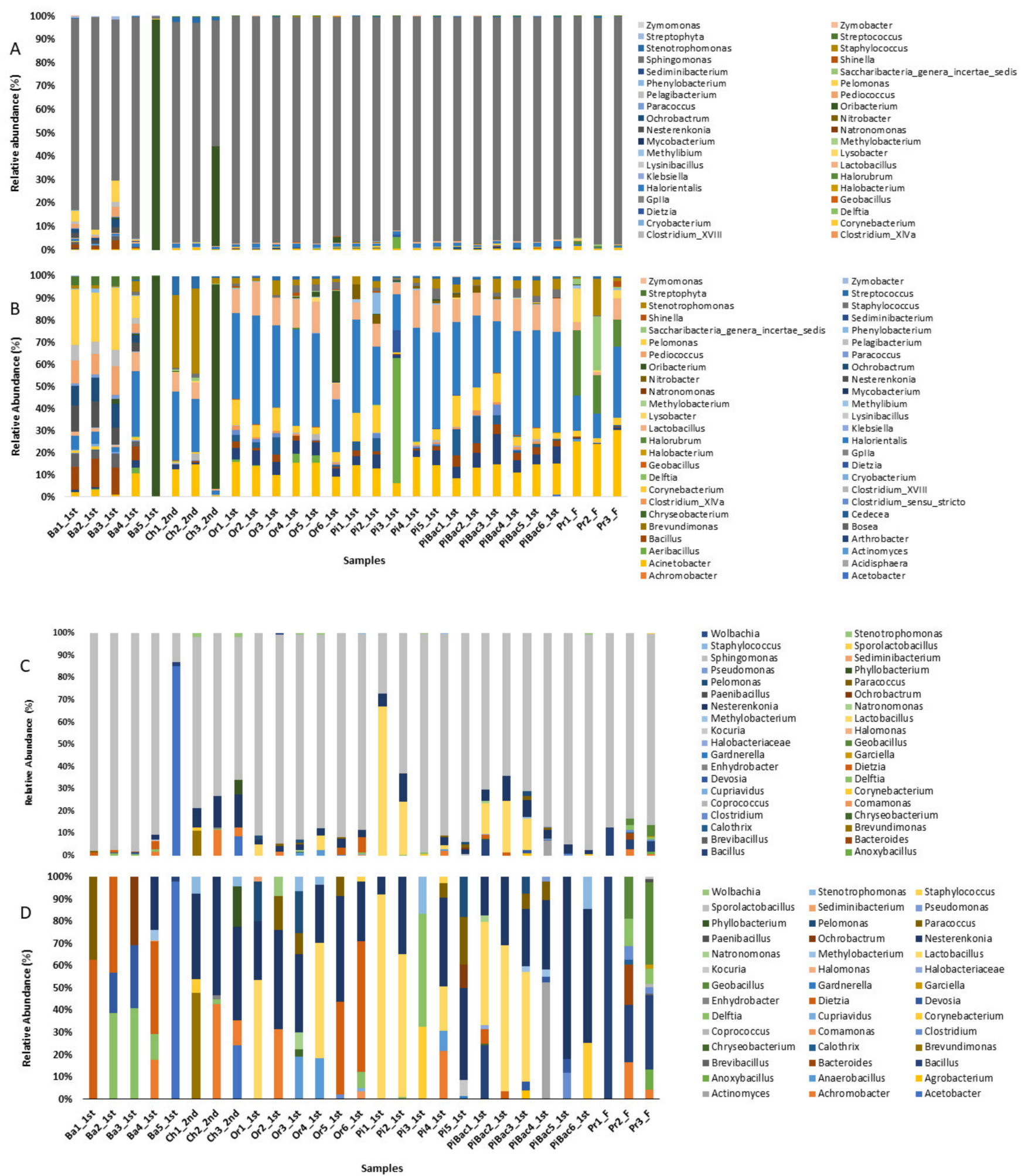

Figure 2. Relative abundance of bacterial diversity at genus level in each fermentation sample using operational taxonomic unit OTU (A) and amplicon sequencing variant ASV-based (C) taxonomic classifications. The dominant genus Sphingomonas was excluded in both OTU (B) and ASV-based (D) taxonomic classifications. 
Table 2. Comparison of pathogenic genera identified using operational taxonomic unit-based and amplicon sequence variant-based clustering approaches and their association with fermentation processes. The symbol $\checkmark$ indicates the presence of the genus, whereas $\times$ represents the absence.

\begin{tabular}{ccccccc}
\hline \multirow{2}{*}{ Genus Names } & \multicolumn{2}{c}{ 1st Stage } & \multicolumn{2}{c}{ 2nd Stage } & \multicolumn{2}{c}{ Final Product } \\
\cline { 2 - 7 } & OTU & ASV & OTU & ASV & OTU & ASV \\
\hline Acinetobacter & $\checkmark$ & $\times$ & $\checkmark$ & $\times$ & $\checkmark$ & $\times$ \\
Bacillus & $\checkmark$ & $\checkmark$ & $\checkmark$ & $\times$ & $\checkmark$ & $\checkmark$ \\
Staphylococcus & $\checkmark$ & $\checkmark$ & $\checkmark$ & $\times$ & $\checkmark$ & $\times$ \\
Dietzia & $\checkmark$ & $\checkmark$ & $\times$ & $\times$ & $\times$ & $\times$ \\
Clostridium & $\checkmark$ & $\checkmark$ & $\checkmark$ & $\times$ & $\checkmark$ & $\checkmark$ \\
Klebsiella & $\checkmark$ & $\times$ & $\checkmark$ & $\times$ & $\times$ & $\times$ \\
Mycobacterium & $\checkmark$ & $\times$ & $\times$ & $\times$ & $\times$ & $\times$ \\
Ochrobactrum & $\checkmark$ & $\checkmark$ & $\times$ & $\times$ & $\times$ & $\times$ \\
Chryseobacterium & $\checkmark$ & $\checkmark$ & $\times$ & $\times$ & $\checkmark$ & $\times$ \\
Stenotrophomonas & $\checkmark$ & $\checkmark$ & $\checkmark$ & $\checkmark$ & $\checkmark$ & $\times$ \\
Streptococcus & $\checkmark$ & $\times$ & $\checkmark$ & $\times$ & $\checkmark$ & $\times$ \\
Gardnerella & $\times$ & $\checkmark$ & $\times$ & $\times$ & $\times$ & $\times$ \\
Pseudomonas & $\times$ & $\checkmark$ & $\times$ & $\times$ & $\times$ & $\times$ \\
\hline
\end{tabular}

\subsection{Change in Bacterial Community Composition Concerning Fermentation Processes} and Products

The changes in bacterial diversity and abundance at the genus level in the samples from different fermentation stages and the final fermentation products were examined using STAMP software. The analysis revealed that the abundance of Dietzia, Devosia, Lactobacillus, Nesterenkonia, Paracoccus, and Pelomonas was significantly different between the first and second stages of fermentation with the abundance of most genera enriched in the first stage of fermentation (Figure 3). Among these genera, Lactobacillus and Nesterenkonia were the predominant genera in the first and second stages of fermentation, respectively. Dietzia, Devosia, Lactobacillus, Nesterenkonia, Paracoccus, Pelomonas, and Stenotrophomonas were significantly enriched in the first stage of fermentation and the final product. However, the mean proportion of these genera markedly decreased in the final product. Moreover, the comparative analysis of bacterial composition revealed that the abundance of only one genus (Nesterenkonia) was significantly different between the second stage of fermentation and the final product. This genus was predominant in the second stage of fermentation, whereas the mean proportion of this genus decreased in the final product. 


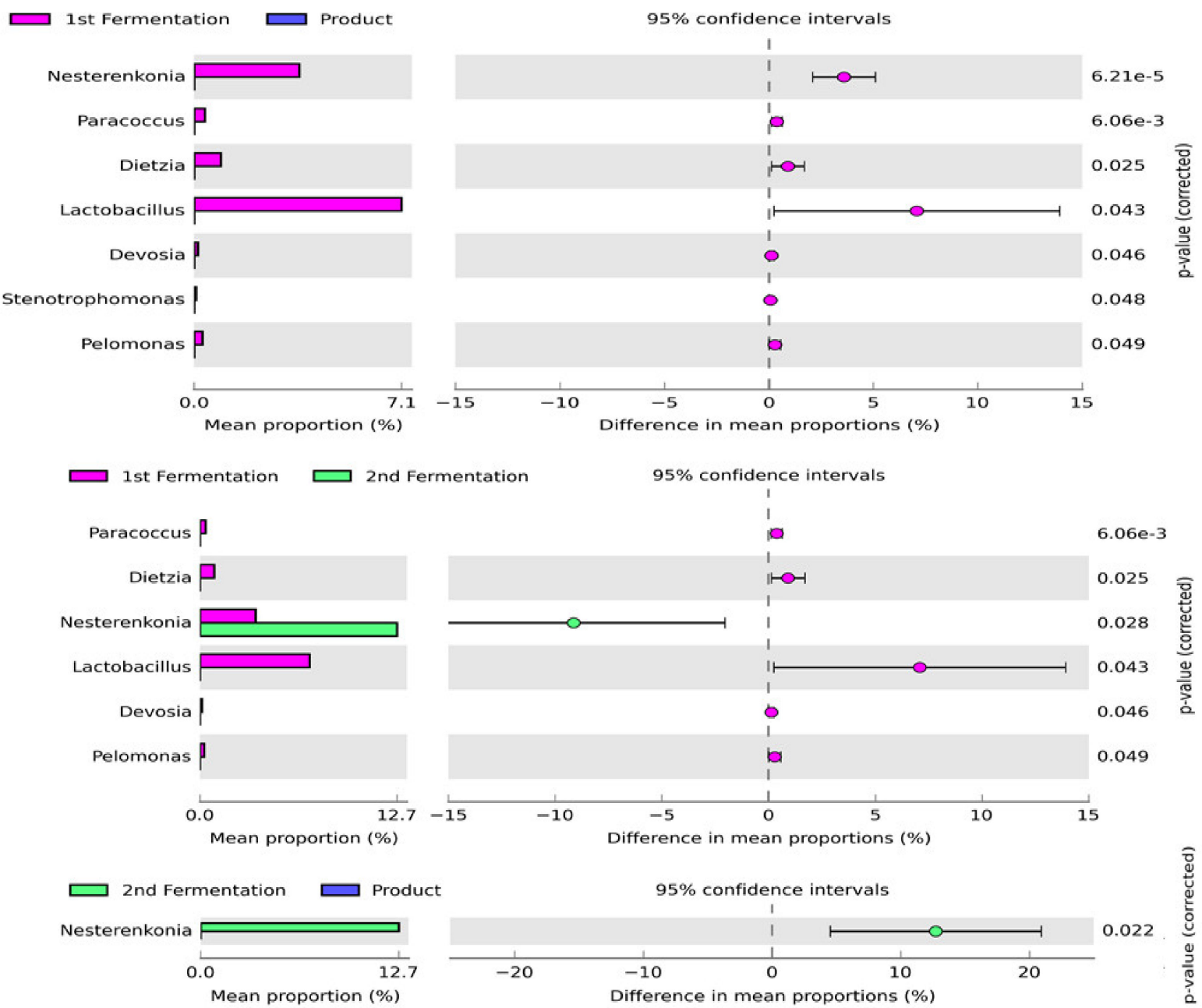

Figure 3. Enriched bacterial genera across the fermentation stages and in the final product. The left panel of these figures shows the abundance of differentially enriched bacterial genera. The right panel represents the significant difference at $p<0.05$. The middle panel indicates the mean proportion of differentially enriched bacterial genera at a $95 \%$ confidence interval.

\subsection{Prediction of Functional Pathways Based on $16 S$ rRNA Gene Metagenomic Data in Fermentation Stages and Final Products}

Functional prediction analysis revealed $5322 \mathrm{KOs}$, which were grouped into 155 KEGG pathways (level 3). These data were further processed to filter the low variant and extremely low abundant pathways ( $<4$ abundance) using MicrobiomAnalyst. In total, 125 high-quality level 3 KEGG pathways were obtained. The level 2 KEGG pathways were presented in the form of a bubble plot for clear visualization and interpretation (Figure 4). These pathways are mainly associated with six major functional categories (KEGG level 1), namely cellular processes $(4.8 \%)$, environmental information processing $(3.2 \%)$, genetic information processing $(9.6 \%)$, human disease $(0.8 \%)$, metabolism $(78.4 \%)$, and organismal systems $(3.2 \%)$. Additionally, the level 2 KEGG pathways related to metabolism, such as carbohydrate, amino acid, cofactor, and vitamin metabolic pathways, were the significant predominant functions in each sample with a minimum relative abundance of $13 \%$. Among the genetic information processing pathways, replication and repair pathway were significantly predominant with a minimum relative abundance of $11.5 \%$ in all fermentation samples. Only one crucial human disease pathway associated with Staphylococcus infection was detected in all samples with a maximum relative abundance of $0.6 \%$. 


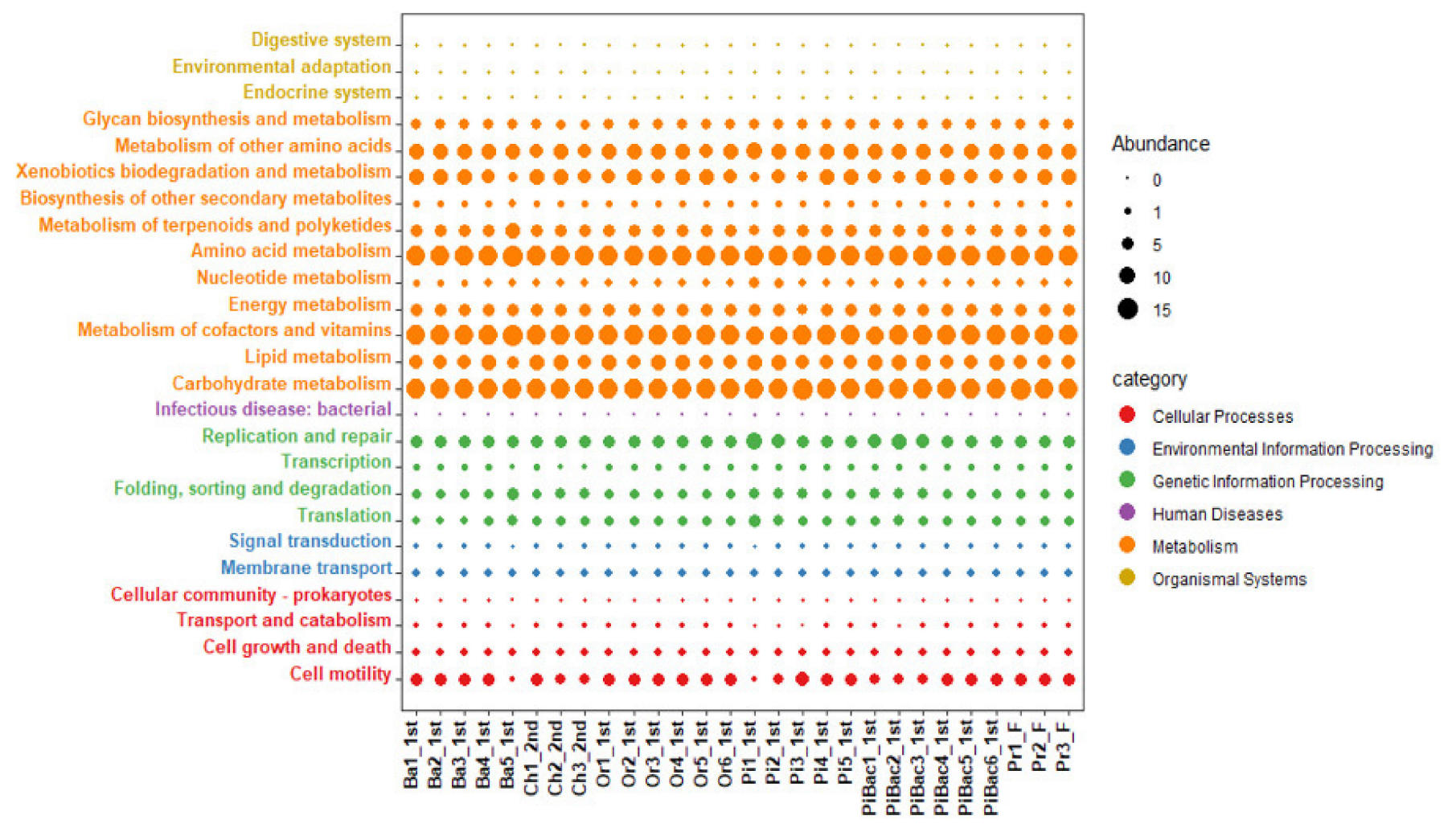

Figure 4. Bubble plot of predicted functions across the fermented fruit samples showing Kyoto Encyclopedia of Genes and Genomes (KEGG) pathway level 2 in different colors on the $Y$-axis belongs to the first category KEGG pathways (legend at right below). The sample names are shown on the $X$-axis. The size and color of the bubble indicate the relative abundance of pathways present in each sample.

The STAMP software was used to further investigate changes in the metabolic functions, especially during the fermentation stages and in the final product (Figure 5). The comparative analysis revealed that six predicted metabolic functions (environmental adaptation, infectious disease, endocrine system, metabolism of other amino acids, and glycan biosynthesis and metabolism) were significantly enriched $(p<0.05)$ in the samples from the first and second stages of fermentation. However, environmental adaptation and infectious disease were significantly abundant in both stages of fermentation. Moreover, the mean proportion of all significant functions decreased in the second stage of fermentation. Glycan biosynthesis and metabolism, translation, replication and repair, nucleotide metabolism, environmental adaptation, cell growth and death, and folding, sorting, and degradation were significantly enriched between the first stage of fermentation and the final product.

Similarly, the mean proportion of all enriched pathways decreased at the end of fermentation. Additionally, metabolism of terpenoids and polyketides, amino acid metabolism, and membrane transport were significantly enriched in the second stage and final products of fermentation. Furthermore, the abundance of these pathways in the final product of fermentation was lower than that in the second stage of fermentation. 

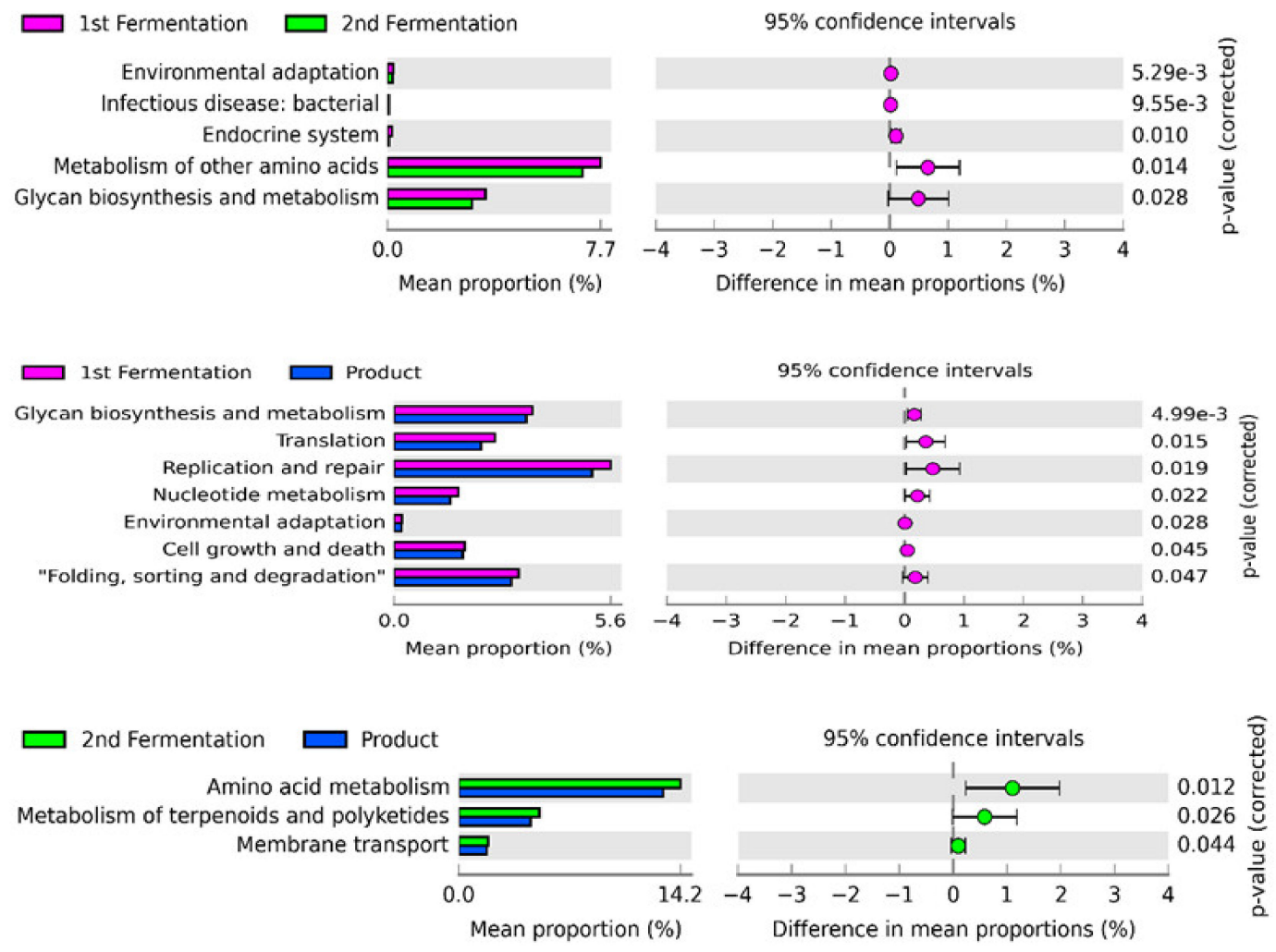

Figure 5. Enriched predicted functions across the fermentation stages and product. The left panel of these figures shows the abundance of differentially predicted functions. The right panel represents the significant difference at $p<0.05$. The middle panel indicates the mean proportion of differentially predicted functions at a $95 \%$ confidence interval.

\subsection{Correlation between the Bacterial Fermentation Community and Predicted Functional Profiles Based on $16 S$ rRNA Amplicon}

Pearson correlation analysis was performed to further explore the correlation between the bacterial community and predicted functions and to identify the significant difference between bacterial taxa at the genus level and the $\mathrm{KO}$ at level 2. The differences were considered significant and highly significant at $p<0.05$ and $<0.01$, respectively (Figure 6).

The correlation analysis revealed that among all the observed bacterial taxa, Sphingomonas was the only predominant genus that was significantly correlated $(p<0.01)$ with all the 25 predicted functions related to fermentation processes and products. Additionally, Paracoccus and Pelomonas were significantly and positively correlated $(p<0.05)$ with only the endocrine system. In contrast, most bacterial taxa, except Acetobacter, were negatively and non-significantly correlated with all predicted functions. Acetobacter was significantly and negatively correlated $(p<0.05)$ with the endocrine system. Similarly, Gardnerella and Kocuria were significantly and positively correlated $(p<0.05)$ with all predicted functions, except cell mobility, transport and co-metabolism, and infectious disease. Additionally, Deleftia, which was the fourth most predominant bacterial taxa, was significantly and positively correlated with more than $50 \%$ of the metabolic pathways $(p<0.01)$. In contrast, only Lactobacillus was significantly and negatively correlated $(p<0.05)$ with cell mobility, cellular community-prokaryote, signal transduction, and digestive system pathways.

None of the identified genera, except Sphingomonas, were significantly and positively correlated with the predicted pathway related to infectious disease, which was the focus of this study. However, Staphylococcus, a potential pathogen, was not significantly and positively correlated with the pathways related to infectious diseases. Staphylococcus was significantly and positively correlated with the endocrine system. 


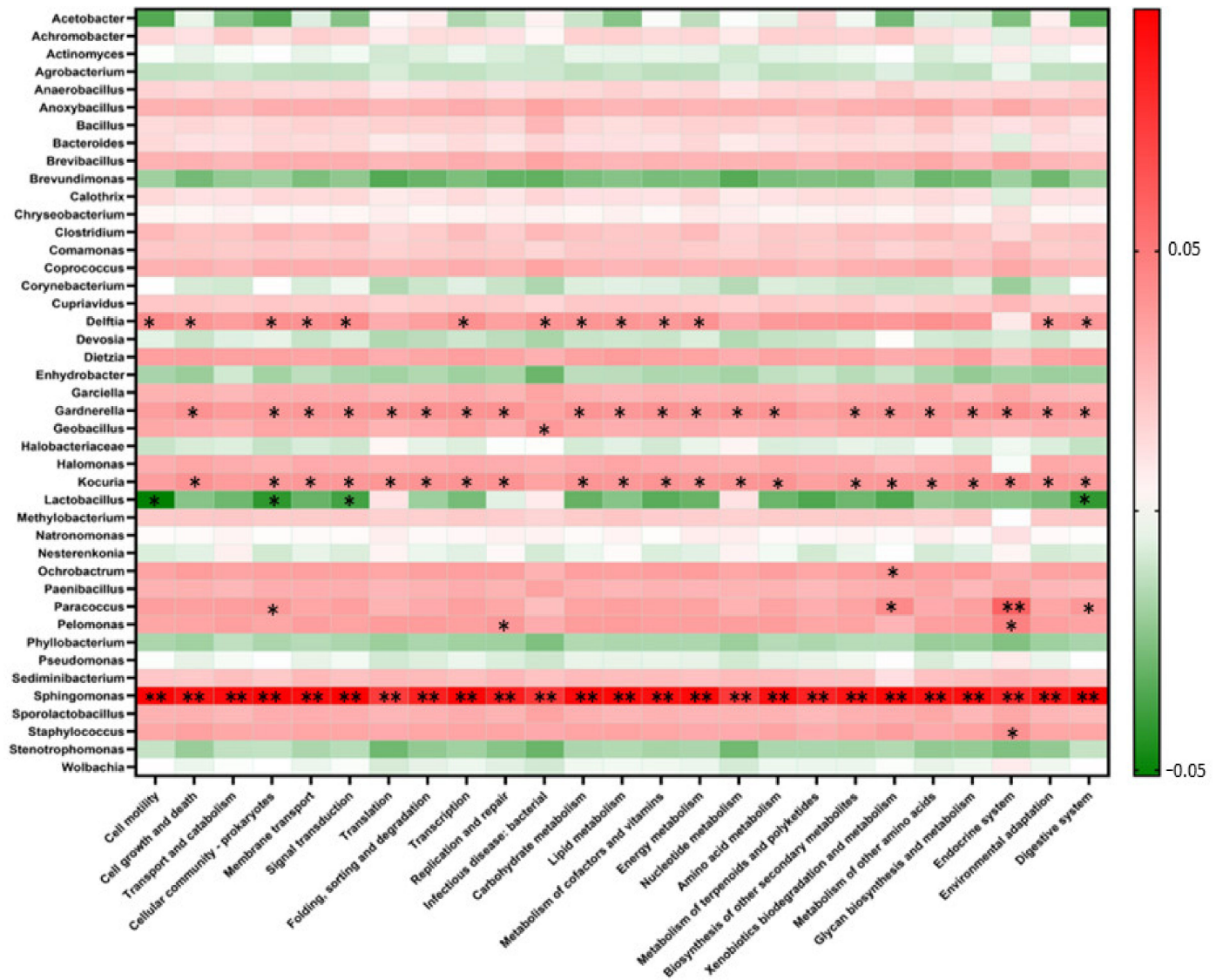

Figure 6. Correlation between the bacterial fermentation community and predicted functional profiles based on $16 \mathrm{~S}$ rRNA amplicon. Pearson correlation analysis heatmap constructed for each pairwise comparison between level 2 Kyoto Encyclopedia of Genes and Genomes (KEGG) pathways and bacterial taxa at the genus level. The positive and negative correlations are indicated in red and green colors, respectively. The correlation was considered significant at ${ }^{* *} p<0.01$ and $* p<0.05$.

\subsection{Confirmation/Validation of Potential Pathogens in Fermentation Stages and Products Using PCR}

The 16S rRNA amplicon sequencing using ASV and OTU based taxonomic classifications revealed 13 genera belonging to potential opportunistic pathogens associated with the fermentation stages and the final product. To confirm their presence or absence in the fermentation stages and product we targeted the following 13 bacterial species using PCR: Acinetobacter baumannii, Bacillus cereus sensu lato s.l, Clostridium difficile, Chryseobacterium indologenes, Dietzia maris, Gardnerella vaginalis, Klebsiella pneumoniae, Pseudomonas aeruginosa, nontuberculous mycobacteria, Ochrobactrum anthropi, Staphylococcus aureus, Streptococcus pneumoniae, and Stenotrophomonas. The PCR analysis revealed absence of all these pathogens except S. aureus was detected only in the Pi4_1st sample, which was from the first fermentation stage. The genus Staphylococcus was also detected in Pi4_1st sample in 16S amplicon sequencing analysis.

\section{Discussion}

Globally, fermented foods are consumed as ready-to-eat foods. However, the lack of microbial characterization of these food products has raised consumer concerns for food safety, which has led to decreased consumption of these essential food items [50]. Modern high-throughput sequencing identifies only the functional and beneficial microbial diversity of fermented products. Therefore, this study investigated the bacterial diversity and identified potential pathogens in different steps of fermentation and their products using high-throughput sequencing approaches and powerful modern bioinformatics. For 
improved representation of bacterial composition containing opportunistic pathogens, $16 \mathrm{~S}$ amplicon sequencing analysis was performed using OTU (USEARCH)-based and ASV (Qiime2)-based taxonomic classification approaches. The OTU-based clustering approach (cut-off value of 97\%) yielded 53 classifiable genera from 785,339 sequence reads, whereas ASV-based clustering (cut-off value of 97) yielded 43 classifiable genera from 193,323 sequence reads. The number of genera identified using ASV-based clustering in this study was consistent with the results of a previous study, which reported 43 genera in Jiaosu (fermented fruit and vegetable juice) using OTU-based clustering UCLUST (cut-off value of $98.6 \%$ [ [51]. The differences in the classification of bacterial genera between these two clustering approaches can be attributed to an increased resolution of ASV-based clustering to rectify the potential sequence errors. In contrast, minimal filtration leads to low-quality sequence reads in the OTU-based clustering approach, which results in a false representation of taxonomic units. Previous studies using different denoising tools and approaches reported a higher number of OTUs than those using ASV-based approaches [52-54]. This can explain the genera missed in the ASV-based clustering approach.

Among the genera detected in both clustering approaches, Sphingomonas was the predominant bacterial genus in almost $90 \%$ of samples and accounted for $80-85 \%$ of all identified bacteria. Other studies have reported that Sphingomonas is associated with pulque fermentation ( $80 \%$ ) and that this was the predominant genus in fermented products of fruit $(78 \%)$ among all identified genera. Sphingomonas, which is considered to be the indigenous flora of soil and plants [55] and is mainly present on the surface of fruits and vegetables, confers resistance and promotes plant growth [53]. A recent study on the fermentation of pickles based on vegetables dehydrated with smoke demonstrated that Sphingomonas was the major bacterial genus in the final product [4]. Additionally, the two taxonomic classification approaches yielded two different genera. Oribacterium was the second most predominant genus in the OTU-based clustering, whereas Nesterenkonia was the predominant genus in ASV-based clustering. Oribacterium and Nesterenkonia are frequently associated with the surface of fruits, water, and soil. A previous study also reported that these two approaches yield different taxonomic levels, which explains the difference in the discriminatory power of these approaches [56]. Moreover, among all the identified genera, 13 low abundant genera were potential opportunistic pathogens. Most of these genera are predominant or rare flora of fruits, vegetables, and skin and are associated with environmental contamination [57]. Similarly, other studies have reported the presence of most of these opportunistic pathogens in the starter cultures and in different stages and products of various fermentation processes, such as cereals, sausages, vegetables, cheese, and fermented fish [58,59]. The contamination of these genera mainly depends on the manufacturing conditions, such as the time-temperature profile, water activity, and $\mathrm{pH}$ applied for fermentation [13]. Other factors that contribute to contamination from pathogenic genera include microbial deterioration and fermented food safety issues, including insufficient sanitation, starter culture quality, and raw material quality [15]. These findings suggest that various factors must be considered to prevent the risk of opportunistic pathogens.

The change in microbial communities during fermentation must be considered to understand the functionality of beneficial microorganisms and the survival of opportunistic pathogens. The results of this study demonstrated that most differentially abundant genera, such as Paracoccus, Dietzia, Devosia, and Pelomonas were significantly enriched in the samples from the first stage of fermentation, whereas their abundance significantly decreased in the samples from the second stage of fermentation. Similarly, Dietzia, Devosia, Lactobacillus, Nesterenkonia, Paracoccus, Pelomonas, and Stenotrophomonas were enriched in the samples from the first stage of fermentation but their abundance significantly decreased in the final product. The increased abundance of these bacteria at the initial stages of fermentation can be attributed to the availability of sufficient nutrients and the suitability of environmental conditions, which promote the proliferation and growth of these bacteria. However, these nutrients are depleted and the culture conditions are unfavorable as the fermentation 
progresses due to decreased $\mathrm{pH}$ caused by organic acid producers, such as Lactobacillus. These changes decrease the abundance of other sensitive microorganisms [57]. Additionally, Lactobacillus was the most abundant genus in the first stage of fermentation, whereas Nesterenkonia was the abundant genus in the second stage of fermentation. Lactobacillus and Nesterenkonia are beneficial for the fermentation of sugars, which leads to acid production and consequently decreased $\mathrm{pH}$ levels. Compared with those at the second stage of fermentation, the production of organic acids was upregulated and the $\mathrm{pH}$ level was lower in the final product as shown in Supplementary Table S2. Lactobacillus is a vital probiotic that produces several types of short-chain fatty acids, which are beneficial to human health [60].

To examine the role of microorganisms during fermentation, PICRUSt2 was used to analyze the 16S rRNA sequencing data. Six major functional categories (KEGG level 1) (metabolism, cellular processes $(4.8 \%)$, environmental information processing $(3.2 \%)$, genetic information processing $(9.6 \%)$, human disease $(0.8 \%)$, metabolism $(78.4 \%)$, and organismal systems (3.2\%)) were significantly associated with the fermentation process. The upregulated function of metabolism suggested the active involvement of microorganisms during fermentation. These results are consistent with those of previous studies, which reported that metabolism was the predominant pathway among the six main KEGG pathways [61,62]. Among the subcategories (KEGG level 2) of these major pathways, carbohydrate, amino acid, cofactor, and vitamin metabolic pathways were the predominant pathways and constituted $13 \%$, while replication and repair functions constituted $11.5 \%$. These inferred functions play an essential role in energy supply, biosynthesis of cellular components, and improving the flavor, taste, and nutritional value of the fermented products [63].

Additionally, the metabolism of other amino acids and glycan biosynthesis and metabolism were the predominant functions between the first and second stages of fermentation. Most functions were significantly enriched in the first stage of fermentation. The functions related to bacterial growth and proliferation, such as replication and repair were the dominant function in the final product. However, amino acid metabolism and metabolism of terpenoids and polyketides were significantly enriched in the second stage and the final product of fermentation. The mean proportion of all significantly enriched pathways decreased in the following order: first stage $>$ second stage $>$ product of fermentation. These results suggest that the bacterial community has varied metabolic capacity and preferential functions in each stage of the fermentation process depending on the fermentation environment. The decline in the mean proportion of these significantly enriched pathways during the fermentation process might be due to the enzymatic activity of various microorganisms. Alternatively, the decline in the number of potential microorganisms responsible for the production of these predicted functions may also contribute to the downregulation of these functions. A similar pattern of decline in the mean proportion of bacterial community was observed in significant microbial shift analysis with the passage of the fermentation process. However, for further confirmation of the presence of these bacterial metabolites and their potential role, we strongly suggest using metabolomics and transcriptomic analyses.

Bacterial function prediction revealed only one crucial predicted human disease pathway associated with Staphylococcus infection (KEGG level 3) was detected in all samples with a maximum relative abundance of $0.6 \%$. This proportion is consistent with the results of PCR analysis but less than that reported in the recent study on Chinese traditional fermented food reports. Approximately $2 \%$ of bacterial genes in the fermentation process were associated with the human disease, which indicated pathogenic contamination or enrichment during the fermentation process. However, further categories of KEGG pathways and microbes associated with the human disease were not reported in their study [64]. Moreover, S. aureus was only detected in the pineapple sample at the first stage of fermentation in ASV-based taxonomic classification and PCR validation assay. 
Pearson correlation analysis was performed to examine the correlation between predicted functions and bacterial community of fermentation. The analysis revealed that Sphingomonas was correlated with all the predicted functions as this was the predominant genus in fermentation samples and accounted for $80-85 \%$ of all observed genera. Additionally, Sphingomonas can metabolize various carbon compounds and toxic compounds that are present in low concentrations of nutrients [65]. Additionally, Delftia and Gardnerella were significantly and positively correlated $(p<0.05)$ with most of the observed functions. In contrast, Lactobacillus was significantly and negatively correlated with cell mobility, cellular community-prokaryotes, signal transduction, and digestive system. Delftia and Gardnerella, which are soilborne bacteria, are commonly found on the surface of fruits. A previous study has demonstrated that these bacteria can metabolize various carbon sources and are correlated with the fermentation processes [66]. Additionally, a significant positive correlation between genus Staphylococcus and infectious disease was not observed. However, this bacterium was significantly and positively correlated with the endocrine system. This may be due to the limited number of sequences $(0.6 \%)$ representing this particular function associated with infectious disease. A previous study demonstrated that differences in the relative abundance of metagenomic data and PICRUSt inferences might not allow an accurate representation of functional traits [67]. Thus, these differences in the functional prediction must be mitigated by increasing the metagenomic sequencing depth.

In this study, the $16 \mathrm{~S}$ rRNA amplicon was sequenced by targeting the V3-V4 regions of DNA for bacterial identification. The identification of bacteria at the species level based on NGS of 16S rRNA targeting the specific conserved regions of DNA is not reliable as it can yield false-positive results [68]. However, the application of NGS for bacterial identification is reliable up to the genus level. Hence, NGS is the most commonly used robust approach to study microbial diversity in different environmental niches. In this study, 13 species of representative opportunistic bacterial pathogens among the taxonomically identified genera were validated. However, these genera mostly comprised non-pathogenic species. Several studies have demonstrated that the accuracy of PCR assays using appropriate primers for bacterial identification at the species level [69,70]. The PCR analysis revealed that only S. aureus was detected in one sample. This indicated that human pathogenic species are not the predominant species in the fermented samples. In this study, the fermented samples were obtained from fruits associated with soil environments. Soil microbes are not considered to be pathogenic. In the genera Acinetobacter and Bacillus, A. calcoaceticus, A. johnsonii, B. subtilis, and B. megaterium are commonly detected in plants and soil [71]. C. difficile is the major pathogenic species in the genus Clostridium. However, more than 250 species have also been reported to be beneficial to human health [72] Although S. aureus was detected in the Pi4_1st sample in the PCR assay, it was not detected in the sample from the second stages and the final product of fermentation. Therefore, this hybrid method could ensure that the pathogen was inactive in the fermentation process and the final fermentation product did not contain pathogenic microorganisms. Additionally, the genus Staphylococcus was identified in the fermentation stages and the final product using OTU-based taxonomical classification. However, Staphylococcus was only identified in the first stage of fermentation using the ASV-based clustering approach. Hence, we propose that compared with the PCR assay, the ASV-based taxonomical classification analysis is more suitable for pathogen prediction.

\section{Conclusions}

This study revealed the correlation between bacterial community composition and functional characteristics during the two stages of fruit fermentation and the final product using high-throughput sequencing and powerful statistical tools. Sphingomonas was predominantly detected during all fermentation stages and in the final product. Additionally, Lactobacillus was significantly enriched with a higher abundance in the 1st stage of fermentation, whereas Nesterenkonia was significantly enriched with a higher abundance in the 2nd stage of fermentation. Functional prediction revealed carbohydrate metabolism was the 
most abundant metabolic pathway throughout the fermentation process. Moreover, Staphylococcus aureus associated with the KEGG human disease pathway was not significantly correlated with opportunistic bacterial pathogenic genera. To confirm the presence of opportunistic bacterial pathogens, PCR assays were performed which revealed absence of these opportunistic pathogens in different fermentation stages or the final products, except in one sample from the first stage of fermentation. Thus, the hybrid method employed in this study can be used to verify the bacterial safety and functions of microorganisms during fermented food production and avoid random detection of opportunistic bacterial pathogens. This hybrid method could provide an effective and high-resolution determinant of the types of bacteria and their functional profile influencing the properties of fermented foods and ensure that no harmful bacteria are present in the final fermentation products.

Supplementary Materials: The following are available online at https:/ / www.mdpi.com/article/10 $.3390 /$ microorganisms9081574/s1, Table S1: The PCR conditions for the identification of suspected pathogens, Table S2: Chemical analysis of fermented fruits from the 2nd stage of fermentation and final products, Table S3: The sequence good's coverage at the minimum sequence depth associated with the fermented samples. Figure S1: Library size overview (A) and alpha rarefaction plot (B) among the fermentation stages and the final products.

Author Contributions: Conceptualization, B.H., B.-M.H., I.-T.C. and J.-S.C.; methodology, J.R., S.K. and T.-H.C.; software, B.H., S.K. and J.-S.C.; validation, M.W.Y.C., I.-T.C., J.-S.C. and B.-M.H.; formal analysis, B.H. and J.-S.C.; investigation, T.-H.C., B.H. and I.-T.C.; resources, J.-S.C. and B.-M.H.; data curation, J.R., B.H. and M.W.Y.C.; writing-original draft preparation, B.H., I.-T.C. and J.-S.C.; writing-review and editing, B.-M.H.; visualization, B.H. and M.W.Y.C.; supervision, B.H., I.-T.C. and J.-S.C.; project administration, B.-M.H.; funding acquisition, I.-T.C. and B.-M.H. All authors have read and agreed to the published version of the manuscript.

Funding: This research was funded by the Taiwan Enzyme Village Co., Ltd. Chiayi, Taiwan, the Ministry of Science and Technology, Taiwan (MOST 108-2116-M-194-005 \& 108-2811-M-194-507), the Ditmanson Medical Foundation Chia-Yi Christian Hospital-National Chung Cheng University Joint Research Program, CYCH-CCU Joint Research Program (CYCH-CCU-2021-05).

Data Availability Statement: In a rigorously anonymous form, raw sequencing data have been deposited in the NCBI depository under the following ID: SUB9829072.

Acknowledgments: We are very thankful for the sample collection and background information provided by the Laboratory Department of Taiwan Enzyme Village Co., Ltd. Chiayi, Taiwan (https: / / enzyvillage.com/, accessed on 5 February 2021).

Conflicts of Interest: The authors declare that they have no conflict of interest.

\section{References}

1. Battcock, M. Fermented Fruits and Vegetables: A Global Perspective; Food \& Agriculture Organization: Rome, Italy, 1998.

2. Hampton, J.; Tang, C.; Jayasree Subhash, A.; Serventi, L. Assessment of pear juice and puree as a fermentation matrix for water kefir. J. Food Process. Preserv. 2021, 45, e15223. [CrossRef]

3. Swain, M.R.; Anandharaj, M.; Ray, R.C.; Rani, R.P. Fermented fruits and vegetables of Asia: A potential source of probiotics. Biotechnol. Res. Int. 2014, 2014, 250424. [CrossRef]

4. Stoll, D.A.; Wafula, E.N.; Mathara, J.M.; Trierweiler, B.; Kulling, S.E.; Huch, M. Fermentation of African nightshade leaves with lactic acid bacterial starter cultures. Int. J. Food Microbiol. 2021, 342, 109056. [CrossRef]

5. Shah, N.N.; Singhal, R.S. 3-Fermented Fruits and Vegetables. In Current Developments in Biotechnology and Bioengineering; Pandey, A., Sanromán, M.Á., Du, G., Soccol, C.R., Dussap, C.-G., Eds.; Elsevier: Amsterdam, The Netherlands, 2017 ; pp. 45-89.

6. Adams, M.; Mitchell, R. Fermentation and pathogen control: A risk assessment approach. Int. J. Food Microbiol. 2002, 79, 75-83. [CrossRef]

7. Sahu, L.; Panda, S. Innovative Technologies and Implications in Fermented Food and Beverage Industries: An Overview. In Innovations in Technologies for Fermented Food and Beverage Industries; Springer: Berlin/Heidelberg, Germany, 2018 ; pp. 1-23.

8. Ae Kim, S.; Jin Park, H.; Jin Cho, T.; Suk Rhee, M. Toxic Potential of Bacillus cereus isolated from fermented alcoholic beverages. Food Res. Int. 2020, 109361. [CrossRef] [PubMed]

9. Cousin, F.J.; Le Guellec, R.; Schlusselhuber, M.; Dalmasso, M.; Laplace, J.-M.; Cretenet, M. Microorganisms in fermented apple beverages: Current knowledge and future directions. Microorganisms 2017, 5, 39. [CrossRef] [PubMed] 
10. Ducic, M.; Blagojevic, B.; Markov, S.; Velicanski, A.; Buncic, S. General patterns of background microbiota and selected bacterial pathogens during production of fermented sausages in Serbia. Food Control 2014, 43, 231-237. [CrossRef]

11. Xiang, H.; Sun-Waterhouse, D.; Waterhouse, G.I.; Cui, C.; Ruan, Z. Fermentation-enabled wellness foods: A fresh perspective. Food Sci. Hum. Wellness 2019, 8, 203-243. [CrossRef]

12. Voidarou, C.; Antoniadou, M.; Rozos, G.; Tzora, A.; Skoufos, I.; Varzakas, T.; Lagiou, A.; Bezirtzoglou, E. Fermentative Foods: Microbiology, Biochemistry, Potential Human Health Benefits and Public Health Issues. Foods 2021, 10, 69. [CrossRef]

13. Mataragas, M.; Bellio, A.; Rovetto, F.; Astegiano, S.; Greci, C.; Hertel, C.; Decastelli, L.; Cocolin, L. Quantification of persistence of the food-borne pathogens Listeria monocytogenes and Salmonella enterica during manufacture of Italian fermented sausages. Food Control 2015, 47, 552-559. [CrossRef]

14. Maillet, A.; Bouju-Albert, A.; Roblin, S.; Vaissié, P.; Leuillet, S.; Dousset, X.; Jaffrès, E.; Combrisson, J.; Prévost, H. Impact of DNA extraction and sampling methods on bacterial communities monitored by $16 \mathrm{~S}$ rDNA metabarcoding in cold-smoked salmon and processing plant surfaces. Food Microbiol. 2021, 95, 103705. [CrossRef]

15. Oguntoyinbo, F.A.; Fusco, V.; Cho, G.-S.; Kabisch, J.; Neve, H.; Bockelmann, W.; Huch, M.; Frommherz, L.; Trierweiler, B.; Becker, B.; et al. Produce from Africa's Gardens: Potential for Leafy Vegetable and Fruit Fermentations. Front. Microbiol. 2016, 7, 981. [CrossRef] [PubMed]

16. D'Alessandro, A.; Zolla, L. We are What We Eat: Food Safety and Proteomics. J. Proteome Res. 2012, 11, 26-36. [CrossRef] [PubMed]

17. Patel, A. Probiotic fruit and vegetable juices-recent advances and future perspective. Int. Food Res. J. 2017, 24, $1850-1857$.

18. Tamang, J.P.; Shin, D.-H.; Jung, S.-J.; Chae, S.-W. Functional Properties of Microorganisms in Fermented Foods. Front. Microbiol. 2016, 7, 578. [CrossRef] [PubMed]

19. Kumbhar, S.; Ghosh, J.; Samudre, S. Microbiological analysis of pathogenic organisms in indigenous fermented milk products. Adv. J. Food Sci. Technol. 2009, 1, 35-38.

20. Marco, M.L.; Sanders, M.E.; Gänzle, M.; Arrieta, M.C.; Cotter, P.D.; De Vuyst, L.; Hill, C.; Holzapfel, W.; Lebeer, S.; Merenstein, D.; et al. The International Scientific Association for Probiotics and Prebiotics (ISAPP) consensus statement on fermented foods. Nat. Rev. Gastroenterol. Hepatol. 2021. [CrossRef] [PubMed]

21. El Sheikha, A.F.; Hu, D.-M. Molecular techniques reveal more secrets of fermented foods. Crit. Rev. Food Sci. Nutr. 2020, 60, 11-32. [CrossRef] [PubMed]

22. Deurenberg, R.H.; Bathoorn, E.; Chlebowicz, M.A.; Couto, N.; Ferdous, M.; García-Cobos, S.; Kooistra-Smid, A.M.; Raangs, E.C.; Rosema, S.; Veloo, A.C. Application of next generation sequencing in clinical microbiology and infection prevention. J. Biotechnol. 2017, 243, 16-24. [CrossRef]

23. López-Campos, G.; Martínez-Suárez, J.V.; Aguado-Urda, M.; López-Alonso, V. Detection, Identification, and Analysis of Foodborne Pathogens. In Microarray Detection and Characterization of Bacterial Foodborne Pathogens; López-Campos, G., MartínezSuárez, J.V., Aguado-Urda, M., López-Alonso, V., Eds.; Springer: Boston, MA, USA, 2012; pp. 13-32.

24. Zhang, W.; Luo, Q.; Zhu, Y.; Ma, J.; Cao, L.; Yang, M.; Wen, P.; Zhang, Z.; He, X. Microbial diversity in two traditional bacterial douchi from Gansu province in northwest China using Illumina sequencing. PLoS ONE 2018, 13, e0194876. [CrossRef]

25. Botta, C.; Cocolin, L. Microbial dynamics and biodiversity in table olive fermentation: Culture-dependent and-independent approaches. Front. Microbiol. 2012, 3, 245. [CrossRef]

26. Cocolin, L.; Alessandria, V.; Dolci, P.; Gorra, R.; Rantsiou, K. Culture independent methods to assess the diversity and dynamics of microbiota during food fermentation. Int. J. Food Microbiol. 2013, 167, 29-43. [CrossRef] [PubMed]

27. Cocolin, L.; Dolci, P.; Rantsiou, K. Biodiversity and dynamics of meat fermentations: The contribution of molecular methods for a better comprehension of a complex ecosystem. Meat Sci. 2011, 89, 296-302. [CrossRef]

28. Brown, E.W.; Gonzalez-Escalona, N.; Stones, R.; Timme, R.; Allard, M.W. The rise of genomics and the promise of whole genome sequencing for understanding microbial foodborne pathogens. In Foodborne Pathogens; Springer: Berlin/Heidelberg, Germany, 2017; pp. 333-351.

29. Jagadeesan, B.; Gerner-Smidt, P.; Allard, M.W.; Leuillet, S.; Winkler, A.; Xiao, Y.; Chaffron, S.; Van Der Vossen, J.; Tang, S.; Katase, M.; et al. The use of next generation sequencing for improving food safety: Translation into practice. Food Microbiol. 2019, 79, 96-115. [CrossRef]

30. Dalmasso, A.; Civera, T.; Pattono, D.; Cardazzo, B.; Bottero, M.T. Characterization of microbiota in Plaisentif cheese by highthroughput sequencing. LWT-Food Sci. Technol. 2016, 69, 490-496. [CrossRef]

31. Zhang, Y.; Skaar, I.; Sulyok, M.; Liu, X.; Rao, M.; Taylor, J.W. The Microbiome and Metabolites in Fermented Pu-erh Tea as Revealed by High-Throughput Sequencing and Quantitative Multiplex Metabolite Analysis. PLoS ONE 2016, 11, e0157847. [CrossRef]

32. Ronholm, J. Editorial: Game Changer-Next Generation Sequencing and Its Impact on Food Microbiology. Front. Microbiol. 2018, 9, 363. [CrossRef]

33. Zheng, W.; Tsompana, M.; Ruscitto, A.; Sharma, A.; Genco, R.; Sun, Y.; Buck, M.J. An accurate and efficient experimental approach for characterization of the complex oral microbiota. Microbiome 2015, 3, 48. [CrossRef] [PubMed]

34. Huang, T.Y.; Hsu, B.M.; Chao, W.C.; Fan, C.W. Plant n-alkane production from litterfall altered the diversity and community structure of alkane degrading bacteria in litter layer in lowland subtropical rainforest in Taiwan. Biogeosciences 2018, 15, 1815-1826. [CrossRef] 
35. Prodan, A.; Tremaroli, V.; Brolin, H.; Zwinderman, A.H.; Nieuwdorp, M.; Levin, E. Comparing bioinformatic pipelines for microbial 16S rRNA amplicon sequencing. PLoS ONE 2020, 15, e0227434. [CrossRef] [PubMed]

36. Parks, D.H.; Tyson, G.W.; Hugenholtz, P.; Beiko, R.G. STAMP: Statistical analysis of taxonomic and functional profiles. Bioinformatics 2014, 30, 3123-3124. [CrossRef]

37. Douglas, G.M.; Maffei, V.J.; Zaneveld, J.; Yurgel, S.N.; Brown, J.R.; Taylor, C.M.; Huttenhower, C.; Langille, M.G.I. PICRUSt2: An improved and customizable approach for metagenome inference. bioRxiv 2020, 672295. [CrossRef]

38. Chong, J.; Liu, P.; Zhou, G.; Xia, J. Using MicrobiomeAnalyst for comprehensive statistical, functional, and meta-analysis of microbiome data. Nat. Protoc. 2020, 15, 799-821. [CrossRef] [PubMed]

39. Chen, T.-L.; Sin, L.-K.; Wu, R.-C.; Shaio, M.-F.; Huang, L.-Y.; Fung, C.-P.; Lee, C.-M.; Cho, W.-L. Comparison of one-tube multiplex PCR, automated ribotyping and intergenic spacer (ITS) sequencing for rapid identification of Acinetobacter baumannii. Clin. Microbiol. Infect. 2007, 13, 801-806. [CrossRef] [PubMed]

40. Aagot, N.; Nybroe, O.; Nielsen, P.; Johnsen, K.J.A.; Microbiology, E. An altered pseudomonas diversity is recovered from soil by using nutrient-poorpseudomonas-selective soil extract media. Appl. Environ. Microbiol. 2001, 67, 5233-5239. [CrossRef] [PubMed]

41. Gallo, S.W.; Ramos, P.L.; Ferreira, C.A.S.; Oliveira, S.D.D. A specific polymerase chain reaction method to identify Stenotrophomonas maltophilia. Mem. Inst. Oswaldo Cruz 2013, 108, 390-391. [CrossRef]

42. Zariffard, M.R.; Saifuddin, M.; Sha, B.E.; Spear, G.T.J.F.I.; Microbiology, M. Detection of bacterial vaginosis-related organisms by real-time PCR for Lactobacilli, Gardnerella vaginalis and Mycoplasma hominis. FEMS Immunol. Med. Microbiol. 2002, 34, 277-281. [CrossRef] [PubMed]

43. Pridgeon, J.W.; Klesius, P.H.; Garcia, J.C. Identification and virulence of C hryseobacterium indologenes isolated from diseased yellow perch (Perca flavescens). J. Appl. Microbiol. 2013, 114, 636-643. [CrossRef]

44. Brakstad, O.G.; Aasbakk, K.; Maeland, J.A. Detection of Staphylococcus aureus by polymerase chain reaction amplification of the nuc gene. J. Clin. Microbiol. 1992, 30, 1654-1660. [CrossRef]

45. Das, S.; Surendran, P.; Thampuran, N. PCR-based detection of enterotoxigenic isolates of Bacillus cereus from tropical seafood. Indian J. Med. Res. 2009, 129, 316-320.

46. Scholz, H.C.; Pfeffer, M.; Witte, A.; Neubauer, H.; Al Dahouk, S.; Wernery, U.; Tomaso, H. Specific detection and differentiation of Ochrobactrum anthropi, Ochrobactrum intermedium and Brucella spp. by a multi-primer PCR that targets the recA gene. J. Med. Microbiol. 2008, 57, 64-71. [CrossRef] [PubMed]

47. Cakmakci, M.L.; Evans, H.; Seidler, R.J. Characteristics of nitrogen-fixing Klebsiella oxytoca isolated from wheat roots. Plant Soil 1981, 61, 53-63. [CrossRef]

48. Greiner, O.; Day, P.J.; Bosshard, P.P.; Imeri, F.; Altwegg, M.; Nadal, D. Quantitative detection of Streptococcus pneumoniae in nasopharyngeal secretions by real-time PCR. J. Clin. Microbiol. 2001, 39, 3129-3134. [CrossRef] [PubMed]

49. Chankhamhaengdecha, S.; Hadpanus, P.; Aroonnual, A.; Ngamwongsatit, P.; Chotiprasitsakul, D.; Chongtrakool, P.; Janvilisri, T. Evaluation of multiplex PCR with enhanced spore germination for detection of Clostridium difficile from stool samples of the hospitalized patients. BioMed Res. Int. 2013, 2013, 875437. [CrossRef] [PubMed]

50. Medina, E.; De Castro, A.; Romero, C.; Ramírez, E.M.; Brenes, M. Safety of fermented fruits and vegetables. In Regulating Safety of Traditional and Ethnic Foods; Elsevier: Amsterdam, The Netherlands, 2016; pp. 355-367.

51. Ma, D.; He, Q.; Ding, J.; Wang, H.; Zhang, H.; Kwok, L.-Y. Bacterial microbiota composition of fermented fruit and vegetable juices (jiaosu) analyzed by single-molecule, real-time (SMRT) sequencing. CyTA-J. Food 2018, 16, 950-956. [CrossRef]

52. Allali, I.; Arnold, J.W.; Roach, J.; Cadenas, M.B.; Butz, N.; Hassan, H.M.; Koci, M.; Ballou, A.; Mendoza, M.; Ali, R.; et al. A comparison of sequencing platforms and bioinformatics pipelines for compositional analysis of the gut microbiome. BMC Microbiol. 2017, 17, 194. [CrossRef]

53. Grützke, J.; Malorny, B.; Hammerl, J.A.; Busch, A.; Tausch, S.H.; Tomaso, H.; Deneke, C. Fishing in the Soup-Pathogen Detection in Food Safety Using Metabarcoding and Metagenomic Sequencing. Front. Microbiol. 2019, 10, 1805. [CrossRef]

54. Nearing, J.T.; Douglas, G.M.; Comeau, A.M.; Langille, M.G. Denoising the Denoisers: An independent evaluation of microbiome sequence error-correction approaches. PeerJ 2018, 6, e5364. [CrossRef]

55. Kim, H.; Nishiyama, M.; Kunito, T.; Senoo, K.; Kawahara, K.; Murakami, K.; Oyaizu, H. High population of Sphingomonas species on plant surface. J. Appl. Microbiol. 1998, 85, 731-736. [CrossRef]

56. Martinson, J.N.; Pinkham, N.V.; Peters, G.W.; Cho, H.; Heng, J.; Rauch, M.; Broadaway, S.C.; Walk, S.T. Rethinking gut microbiome residency and the Enterobacteriaceae in healthy human adults. ISME J. 2019, 13, 2306-2318. [CrossRef]

57. Hao, Y.; Sun, B. Analysis of bacterial diversity and biogenic amines content during fermentation of farmhouse sauce from Northeast China. Food Control 2020, 108, 106861. [CrossRef]

58. Park, K.M.; Kim, H.J.; Jeong, M.C.; Koo, M. Occurrence of Toxigenic Bacillus cereus and Bacillus thuringiensis in Doenjang, a Korean Fermented Soybean Paste. J. Food Prot. 2016, 79, 605-612. [CrossRef]

59. Ezeonu, C.S.; Ezeonu, N.C. Biological Risks Associated with Fermented Diary Products, Fruits, Vegetables and Meat: A Critical Review. Adv. Biotechnol. Microbiol. 2017, 2, 555577. [CrossRef]

60. Qiao, H.; Zhang, L.; Shi, H.; Song, Y.; Bian, C. Astragalus affects fecal microbial composition of young hens as determined by $16 \mathrm{~S}$ rRNA sequencing. AMB Express 2018, 8, 70. [CrossRef]

61. Jeong, S.E.; Chun, B.H.; Kim, K.H.; Park, D.; Roh, S.W.; Lee, S.H.; Jeon, C.O. Genomic and metatranscriptomic analyses of Weissella koreensis reveal its metabolic and fermentative features during kimchi fermentation. Food Microbiol. 2018, 76, 1-10. [CrossRef] 
62. Xie, M.; An, F.; Zhao, Y.; Wu, R.; Wu, J. Metagenomic analysis of bacterial community structure and functions during the fermentation of da-jiang, a Chinese traditional fermented food. LWT 2020, 129, 109450. [CrossRef]

63. Wu, J.; Tian, T.; Liu, Y.; Shi, Y.; Tao, D.; Wu, R.; Yue, X. The dynamic changes of chemical components and microbiota during the natural fermentation process in Da-Jiang, a Chinese popular traditional fermented condiment. Food Res. Int. 2018, 112, $457-467$. [CrossRef]

64. Xie, M.; An, F.; Yue, X.; Liu, Y.; Shi, H.; Yang, M.; Cao, X.; Wu, J.; Wu, R. Characterization and comparison of metaproteomes in traditional and commercial dajiang, a fermented soybean paste in northeast China. Food Chem. 2019, 301, 125270. [CrossRef]

65. Xu, P.; Yu, B.; Li, F.L.; Cai, X.F.; Ma, C.Q. Microbial degradation of sulfur, nitrogen and oxygen heterocycles. Trends Microbiol. 2006, 14, 398-405. [CrossRef]

66. Hsieh, W.-C.; Wada, Y.; Chang, C.-P. Fermentation, biodegradation and tensile strength of poly(3-hydroxybutyrate-co-4hydroxybutyrate) synthesized by Delftia acidovorans. J. Taiwan Inst. Chem. Eng. 2009, 40, 143-147. [CrossRef]

67. Staley, C.; Gould, T.J.; Wang, P.; Phillips, J.; Cotner, J.B.; Sadowsky, M.J. Core functional traits of bacterial communities in the Upper Mississippi River show limited variation in response to land cover. Front. Microbiol. 2014, 5, 414. [CrossRef]

68. Winand, R.; Bogaerts, B.; Hoffman, S.; Lefevre, L.; Delvoye, M.; Van Braekel, J.; Fu, Q.; Roosens, N.H.; De Keersmaecker, S.C.; Vanneste, K. Targeting the 16s rRNA gene for bacterial identification in complex mixed samples: Comparative evaluation of second (illumina) and third (oxford nanopore technologies) generation sequencing technologies. Int. J. Mol. Sci. 2020, 21, 298. [CrossRef] [PubMed]

69. Kim, E.; Yang, S.-M.; Lim, B.; Park, S.H.; Rackerby, B.; Kim, H.-Y. Design of PCR assays to specifically detect and identify 37 Lactobacillus species in a single 96 well plate. BMC Microbiol. 2020, 20, 96. [CrossRef]

70. Martineau, F.; Picard, F.J.; Roy, P.H.; Ouellette, M.; Bergeron, M.G. Species-specific and ubiquitous-DNA-based assays for rapid identification of Staphylococcus aureus. J. Clin. Microbiol. 1998, 36, 618-623. [CrossRef]

71. Hrenovic, J.; Durn, G.; Goic-Barisic, I.; Kovacic, A. Occurrence of an environmental Acinetobacter baumannii strain similar to a clinical isolate in paleosol from Croatia. Appl. Environ. Microbiol. 2014, 80, 2860-2866. [CrossRef]

72. Guo, P.; Zhang, K.; Ma, X.; He, P. Clostridium species as probiotics: Potentials and challenges. J. Anim. Sci. Biotechnol. 2020, 11, 24. [CrossRef] [PubMed] 